\title{
Bertrand Curves of AW( $k)$-Type in the Equiform Geometry of the Galilean Space
}

\author{
Sezai Kızlltuğ ${ }^{1,2}$ and Yusuf Yaylı ${ }^{1,2}$ \\ ${ }^{1}$ Department of Mathematics, Faculty of Arts and Sciences, Erzincan University, 24000 Erzincan, Turkey \\ ${ }^{2}$ Department of Mathematics, Faculty of Sciences, Ankara University, 06000 Ankara, Turkey \\ Correspondence should be addressed to Sezai Kız1ltuğ; skiziltug24@hotmail.com
}

Received 27 October 2013; Accepted 14 February 2014; Published 6 April 2014

Academic Editor: Bernhard Ruf

Copyright (c) 2014 S. Kızltuğ and Y. Yaylı. This is an open access article distributed under the Creative Commons Attribution License, which permits unrestricted use, distribution, and reproduction in any medium, provided the original work is properly cited.

We consider curves of AW( $k$ )-type $(1 \leq k \leq 3)$ in the equiform geometry of the Galilean space $G_{3}$. We give curvature conditions of curves of AW $(k)$-type. Furthermore, we investigate Bertrand curves in the equiform geometry of $G_{3}$. We have shown that Bertrand curve in the equiform geometry of $G_{3}$ is a circular helix. Besides, considering $\mathrm{AW}(k)$-type curves, we show that there are Bertrand curves of weak AW(2)-type and AW(3)-type. But, there are no such Bertrand curves of weak AW(3)-type and AW(2)-type.

\section{Introduction}

A Galilean space may be considered as the limit case of a pseudo-Euclidean space in which the isotropic cone degenerates to a plane. This limit transition corresponds to the limit transition from the special theory of relativity to classical mechanics. On the other hand, Galilean space-time plays an important role in nonrelativistic physics. The fact that the fundamental concepts such as velocity, momentum, kinetic energy, and principles; laws of motion and conservation laws of classical physics are expressed in terms of Galilean space [1]. As it is well known, geometry of space is associated with mathematical group. The idea of invariance of geometry under transformation group may imply that on some spacetimes of maximum symmetry there should be a principle of relativity, which requires the invariance of physical laws without gravity under transformations among inertial systems. Besides, the theory of curves and the curves of constant curvature in the equiform differential geometry of the isotropic spaces $I_{3}^{1}$ and $I_{3}^{2}$ and the Galilean space $G_{3}$ are described in $[2,3]$, respectively. Although the equiform geometry has minor importance related to the usual one, the curves that appear here in the equiform geometry can be seen as generalizations of well-known curves from the above mentioned geometries and therefore could have been of research interest. Many interesting results on curves of
AW $(k)$-type have been obtained by many mathematicians (see [4-7]). For example, in [4], Özgür and Gezgin studied a Bertrand curve of AW $(k)$-type, and furthermore they showed that there was no such Bertrand curve of AW(1)-type and it was of AW(3)-type if and only if it was a right circular helix. In addition they studied weak AW(2)-type and AW(3)type conical geodesic curves in $E^{3}$. Besides, in 3-dimensional Galilean space and Lorentz space, the curves of AW $(k)$-type were investigated by Külahc1 et al. [8] and Külahcı and Ergüt [6], respectively. Kizıltuğ and Yaylı investigated quaternionic AW( $k$-type curves [9]. Also, Kizlltuğ and Yaylı [7] studied curves of AW( $k)$-type in three Lie groups and gave some interesting results.

The purpose of the present paper is to provide $\mathrm{AW}(k)$ type curves in the equiform geometry of the Galilean space $G_{3}$ and provide the properties of Bertrand curve of AW $(k)$ type in the equiform geometry of the Galilean space $G_{3}$.

\section{Preliminaries}

The Galilean space $G_{3}$ is a Cayley-Klein space equipped with the projective metric of signature $(0,0,+,+)$. The absolute figure of the Galilean space consists of an ordered triple $\{w, f, I\}$, where $w$ is the ideal (absolute) plane, $f$ is the line 
(absolute line) in $w$, and $I$ is the fixed elliptic involution of points of $f$.

In the nonhomogeneous coordinates the similarity group $\mathrm{H}_{8}$ has the form

$$
\begin{aligned}
& \bar{x}=a_{11}+a_{12} x, \\
& \bar{y}=a_{21}+a_{22} x+a_{23} y \cos \theta+a_{23} z \sin \theta, \\
& \bar{z}=a_{31}+a_{32} x-a_{23} y \sin \theta+a_{23} z \cos \theta
\end{aligned}
$$

where $a_{i j}$ and $\theta$ are real numbers [10]. In what follows the real numbers $a_{12}$ and $a_{23}$ will play the special role. In particular, for $a_{12}=a_{23}=1$, (1) defines the group $B_{6} \subset H_{8}$ of isometries of the Galilean space $G_{3}$. The Galilean scalar product can be written as

$$
\langle x, y\rangle= \begin{cases}x_{1} x_{2}, & \text { if } x_{1} \neq 0 \text { or } x_{2} \neq 0 \\ y_{1} y_{2}+z_{1} z_{2}, & \text { if } x_{1} \neq 0, x_{2}=0\end{cases}
$$

where $x=\left(x_{1}, y_{1}, z_{1}\right)$ and $y=\left(x_{2}, y_{2}, z_{2}\right)$. It leaves invariant the Galilean norm of the vector $x$ defined by

$$
\|x\|= \begin{cases}x_{1}, & \text { if } x_{1} \neq 0 \\ \sqrt{y_{1}^{2}+z_{1}^{2}}, & \text { if } x_{1}=0\end{cases}
$$

A curve $\alpha: I \subset \mathbb{R} \rightarrow G_{3}$ of the class $C^{\infty}$ in the Galilean space $G_{3}$ is defined by the parameterization

$$
\alpha(s)=(s, y(s), z(s))
$$

where $s$ is a Galilean invariant arc-length of $\alpha$. Then the curvature $\kappa(s)$ and the torsion $\tau(s)$ are given by, respectively,

$$
\begin{aligned}
\kappa(s) & =\sqrt{\ddot{y}(s)^{2}+\ddot{z}(s)^{2}}, \\
\tau(s) & =\frac{\operatorname{det}(\dot{\alpha}(s), \ddot{\alpha}(s), \ddot{\alpha}(s))}{\kappa^{2}(s)} .
\end{aligned}
$$

On the other hand, the Frenet vectors of $\alpha(s)$ in $G_{3}$ are defined by

$$
\begin{aligned}
& t(s)=\dot{\alpha}(s)=(1, \dot{y}(s), \dot{z}(s)), \\
& n(s)=\frac{1}{\kappa(s)} \ddot{\alpha}(s)=\frac{1}{\kappa(s)}(0, \ddot{y}(s), \ddot{z}(s)), \\
& b(s)=\frac{1}{\kappa(s)}(0,-\ddot{z}(s), \ddot{y}(s)) .
\end{aligned}
$$

The vectors $t, n, b$ are called the vectors of tangent, principal normal, and binormal of $\alpha$, respectively. For their derivatives, the following Frenet formula satisfies [10]:

$$
\begin{aligned}
& \dot{t}(s)=\kappa(s) n(s), \\
& \dot{n}(s)=\tau(s) b(s), \\
& \dot{b}(s)=-\tau(s) n(s) .
\end{aligned}
$$

\section{Frenet Formulas in}

\section{Equiform Geometry in $G_{3}$}

Let $\alpha: I \rightarrow G_{3}$ be a curve in the Galilean space $G_{3}$. We define the equiform parameter of $\alpha$ by

$$
\sigma:=\int \frac{1}{\rho} d s=\int \kappa d s,
$$

where $\rho=(1 / \kappa)$ is the radius of curvature of the curve $\alpha$. Then, we have

$$
\frac{d s}{d \sigma}=\rho
$$

Let $h$ be a homothety with the center in the origin and the coefficient $\lambda$. If we put $\widetilde{\alpha}=h(\alpha)$, then it follows

$$
\widetilde{s}=\lambda s, \quad \widetilde{\rho}=\lambda \rho,
$$

where $\tilde{s}$ is the arc-length parameter of $\tilde{\alpha}$ and $\tilde{\rho}$ the radius of curvature of this curve. Therefore, $\sigma$ is an equiform invariant parameter of $\alpha[10]$.

From now on, we define the Frenet formula of the curve $\alpha$ with respect to the equiform invariant parameter $\sigma$ in $G_{3}$. The vector

$$
T=\frac{d \alpha}{d \sigma}
$$

is called a tangent vector of the curve $\alpha$. From (7) and (9) we get

$$
T=\frac{d \alpha}{d s} \frac{d s}{d \sigma}=\rho \cdot \frac{d \alpha}{d s}=\rho \cdot t
$$

We define the principal normal vector and the binormal vector by

$$
N=\rho \cdot n, \quad B=\rho \cdot b .
$$

Then, we easily show that $\{T, N, B\}$ are an equiform invariant orthonormal frame of the curve $\alpha$.

On the other hand, the derivations of these vectors with respect to $\sigma$ are given by

$$
\begin{aligned}
& T^{\prime}=\frac{d T}{d \sigma}=\dot{\rho} T+N, \\
& N^{\prime}=\frac{d N}{d \sigma}=\dot{\rho} N+\rho \tau B, \\
& B^{\prime}=\frac{d B}{d \sigma}=\rho \tau N+\dot{\rho} B .
\end{aligned}
$$

Definition 1. The function $\mathscr{K}: I \rightarrow \mathbb{R}$ defined by

$$
\mathscr{K}=\dot{\rho}
$$

is called the equiform curvature of the curve $\alpha$.

Definition 2. The function $\mathscr{T}: I \rightarrow \mathbb{R}$ defined by

$$
\mathscr{T}=\rho \tau=\frac{\tau}{\kappa}
$$

is called the equiform torsion of the curve $\alpha$. 
Thus, the formula analogous to the Frenet formula in the equiform geometry of the Galilean space has the following form:

$$
\begin{aligned}
T^{\prime} & =\mathscr{K} \cdot T+N, \\
N^{\prime} & =\mathscr{K} \cdot N+\mathscr{T} \cdot B, \\
B^{\prime} & =-\mathscr{T} \cdot N+\mathscr{K} \cdot B .
\end{aligned}
$$

The equiform parameter $\sigma=\int \kappa(s) d s$ for closed curves is called the total curvature, and it plays an important role in global differential geometry of Euclidean space. Also, the function $(\tau / \kappa)$ has been already known as a conical curvature, and it also has interesting geometric interpretation.

Remark 3. Let $\alpha: I \rightarrow G_{3}$ be a curve in the equiform geometry of the Galilean space $G_{3}$. So the following statements are true (see for details $[2,10]$ ).

(i) If $\alpha(s)$ is an isotropic logarithmic spiral in $G_{3}$, then $\mathscr{K}=$ const. $\neq 0$ and $\mathscr{T}=0$.

(ii) If $\alpha(s)$ is an circular helix in $G_{3}$, then $\mathscr{K}=0$ and $\mathscr{T}=$ const. $\neq 0$.

(iii) If $\alpha(s)$ is an isotropic circle in $G_{3}$, then $\mathscr{K}=0$ and $\mathscr{T}=0$.

\section{4. $\mathbf{A W}(k)$-Type Curves in Equiform Geometry in $G_{3}$}

Let $\alpha: I \rightarrow G_{3}$ be a curve in the Galilean space $G_{3}$. The curve $\alpha$ is called a Frenet curve of osculating order 3 if its derivatives $\alpha^{\prime}(s), \alpha^{\prime \prime}(s), \alpha^{\prime \prime \prime}(s), \alpha^{\prime \prime \prime \prime}(s)$ are linearly dependent, and $\alpha^{\prime}(s)$, $\alpha^{\prime \prime}(s), \alpha^{\prime \prime \prime}(s), \alpha^{\prime \prime \prime \prime}(s)$ are no longer linearly independent for all $s \in I$.

Proposition 4. Let $\alpha: I \rightarrow G_{3}$ be a curve in the equiform geometry of the Galilean space $G_{3}$; one has

$$
\begin{aligned}
\alpha^{\prime}(s)= & T(s), \\
\alpha^{\prime \prime}(s)= & \mathscr{K}(s) T(s)+N(s), \\
\alpha^{\prime \prime \prime}(s)= & \left.\mathscr{K}^{\prime}(s)+\mathscr{K}^{2}(s)\right) T(s) \\
& +2 \mathscr{K}(s) N(s)+\mathscr{T}(s) B(s), \\
\alpha^{\prime \prime \prime \prime}(s)= & \left(\mathscr{K}^{\prime \prime}(s)+3 \mathscr{K}(s) \mathscr{K}^{\prime}(s)\right) T(s) \\
& +\left(3 \mathscr{K}^{\prime}(s)+3 \mathscr{K}^{2}(s)-\mathscr{T}^{2}(s)\right) N(s) \\
& +\left(3 \mathscr{K}(s) \mathscr{T}(s)+\mathscr{T}^{\prime}(s)\right) B(s) .
\end{aligned}
$$

Notation. Let us write

$$
\begin{aligned}
N_{1}(s)= & N(s), \\
N_{2}(s)= & 2 \mathscr{K}(s) N(s)+\mathscr{T}(s) B(s), \\
N_{3}(s)= & \left(3 \mathscr{K}^{\prime}(s)+3 \mathscr{K}^{2}(s)-\mathscr{T}^{2}(s)\right) N(s) \\
& +\left(3 \mathscr{K}(s) \mathscr{T}(s)+\mathscr{T}^{\prime}(s)\right) B(s) .
\end{aligned}
$$

Remark 5. $\alpha^{\prime}(s), \alpha^{\prime \prime}(s), \alpha^{\prime \prime \prime}(s), \alpha^{\prime \prime \prime \prime}(s)$ are linearly dependent if and only if $N_{1}(s), N_{2}(s), N_{3}(s)$ are linearly dependent.

As the definition of $\mathrm{AW}(k)$-type curves in [5], we have the following definition.

Definition 6. Curves (of osculating order 3) in the equiform geometry of the Galilean space are given as

(i) of type weak AW(2) if they satisfy

$$
N_{3}(s)=\left\langle N_{3}(s), N_{2}^{*}(s)\right\rangle N_{2}^{*}(s),
$$

(ii) of type weak AW(3) if they satisfy

$$
N_{3}(s)=\left\langle N_{3}(s), N_{1}^{*}(s)\right\rangle N_{1}^{*}(s),
$$

where

$$
\begin{gathered}
N_{1}^{*}(s)=\frac{N_{1}(s)}{\left\|N_{1}(s)\right\|}, \\
N_{2}^{*}(s)=\frac{N_{2}(s)-\left\langle N_{2}(s), N_{1}^{*}(s)\right\rangle N_{1}^{*}(s)}{\left\|N_{2}(s)-\left\langle N_{2}(s), N_{1}^{*}(s)\right\rangle N_{1}^{*}(s)\right\|} .
\end{gathered}
$$

Proposition 7. Let $\alpha: I \rightarrow G_{3}$ be a curve (of osculating order 3 ) in the equiform geometry of the Galilean space $G_{3}$. Then $\alpha$ is of type weak $A W(2)$ if and only if

$$
3 \mathscr{K}(s) \mathscr{T}(s)+\mathscr{T}^{\prime}(s)=0 .
$$

Corollary 8. Let $\alpha: I \rightarrow G_{3}$ be a curve (of osculating order 3 ) in the equiform geometry of the Galilean space $G_{3}$.

(i) If $\alpha$ is an isotropic logarithmic spiral in $G_{3}$, then $\alpha$ is type weak $A W(2)$ curve.

(ii) If $\alpha$ is a circular helix in $G_{3}$, then $\alpha$ is type weak $A W(2)$ curve.

(iii) If $\alpha$ is an isotropic circle in $G_{3}$, then $\alpha$ is type weak $A W(2)$ curve.

Proof. By using Remark 3 and Proposition 7, we have the results.

Proposition 9. Let $\alpha: I \rightarrow G_{3}$ be a curve (of osculating order 3 ) in the equiform geometry of the Galilean space $G_{3}$. If $\alpha$ is of type weak $A W(3)$, then

$$
3 \mathscr{K}^{\prime}(s)+3 \mathscr{K}^{2}(s)-\mathscr{T}^{2}(s)=0
$$

Corollary 10. If $\alpha$ is an isotropic circle in $G_{3}$. Then $\alpha$ is of type weak $A W(3)$ curve.

Proof. It is obvious from Remark 3 and Proposition 9.

Corollary 11. Let $\alpha: I \rightarrow G_{3}$ be a curve (of osculating order 3 ) in the equiform geometry of the Galilean space $G_{3}$. Then there is no isotropic logarithmic spiral or circular helix of type weak $A W(3)$. 
Proof. If $\alpha$ is an isotropic logarithmic spiral or circular helix, then from Remark 3 we have, respectively,

$$
\begin{aligned}
& \mathscr{K}(s)=\text { const. } \neq 0, \quad \mathscr{T}(s)=0, \\
& \mathscr{K}(s)=0, \quad \mathscr{T}(s)=\text { const. } \neq 0 .
\end{aligned}
$$

Substituting (27) and (28) in (26), we get, respectively,

$$
\mathscr{K}^{2}(s)=0, \quad \mathscr{T}^{2}(s)=0 .
$$

Since $\mathscr{K}(s)$ is nonzero constant and $\mathscr{T}(s)$ is nonzero constant, this is impossible, so $\alpha$ is not isotropic logarithmic spiral or circular helix of type weak AW(3).

Definition 12. Curves (of osculating order 3) in the equiform geometry of the Galilean space are given as

(i) of type AW(1) if they satisfy $N_{3}(s)=0$,

(ii) of type $\mathrm{AW}(2)$ if they satisfy

$$
\left\|N_{2}(s)\right\|^{2} N_{3}(s)=\left\langle N_{3}(s), N_{2}(s)\right\rangle N_{2}(s),
$$

(iii) of type $\mathrm{AW}(3)$ if they satisfy

$$
\left\|N_{1}(s)\right\|^{2} N_{3}(s)=\left\langle N_{3}(s), N_{1}(s)\right\rangle N_{1}(s) .
$$

Theorem 13. Let $\alpha: I \rightarrow G_{3}$ be a curve (of osculating order 3 ) in the equiform geometry of the Galilean space $G_{3}$. Then $\alpha$ is of type $A W(1)$ if and only if

$$
\begin{gathered}
3 \mathscr{K}^{\prime}(s)+3 \mathscr{K}^{2}(s)-\mathscr{T}^{2}(s)=0, \\
3 \mathscr{K}(s) \mathscr{T}(s)+\mathscr{T}^{\prime}(s)=0 .
\end{gathered}
$$

Proof. Since $\alpha$ is a curve of type AW(1), we have $N_{3}(s)=0$. Then from (21), we have

$$
\begin{aligned}
&\left(3 \mathscr{K}^{\prime}(s)+3 \mathscr{K}^{2}(s)-\mathscr{T}^{2}(s)\right) N(s) \\
& \quad+\left(3 \mathscr{K}(s) \mathscr{T}(s)+\mathscr{T}^{\prime}(s)\right) B(s)=0 .
\end{aligned}
$$

Furthermore, since $N(s)$ and $B(s)$ are linearly independent, we get

$$
\begin{gathered}
3 \mathscr{K}^{\prime}(s)+3 \mathscr{K}^{2}(s)-\mathscr{T}^{2}(s)=0, \\
3 \mathscr{K}(s) \mathscr{T}(s)+\mathscr{T}^{\prime}(s)=0 .
\end{gathered}
$$

The converse statement is trivial. Hence our theorem is proved.

Corollary 14. If $\alpha(s)$ is an isotropic circle in $G_{3}$, then $\alpha$ is of type $A W(1)$ curve.

Proof. The proof is obvious from Remark 3 and Theorem 13.

Theorem 15. Let $\alpha: I \rightarrow G_{3}$ be a curve (of osculating order 3 ) in the equiform geometry of the Galilean space $G_{3}$. Then $\alpha$ is of type $A W(2)$ if and only if

$$
\begin{gathered}
6 \mathscr{K}^{2}(s) \mathscr{T}(s)-2 \mathscr{K}(s) \mathscr{T}^{\prime}(s)-3 \mathscr{T}(s) \mathscr{K}^{\prime}(s) \\
-\mathscr{T}^{3}(s)-3 \mathscr{K}^{2}(s) \mathscr{T}(s)=0 .
\end{gathered}
$$

Proof. Suppose that $\alpha$ is a Frenet curve of order 3; then from (20) and (21), we can write

$$
\begin{aligned}
& N_{2}(s)=\gamma(s) N(s)+\beta(s) B(s), \\
& N_{3}(s)=\eta(s) N(s)+\delta(s) B(s),
\end{aligned}
$$

where $\gamma, \beta, \eta$, and $\delta$ are differentiable functions. Since $N_{2}(s)$ and $N_{3}(s)$ are linearly dependent, coefficients determinant is equal to zero and hence one can write

$$
\left|\begin{array}{ll}
\gamma(s) & \beta(s) \\
\eta(s) & \delta(s)
\end{array}\right|=0
$$

Here,

$$
\begin{gathered}
\gamma(s)=2 \mathscr{K}(s), \quad \beta(s)=\mathscr{T}(s), \\
\eta(s)=3 \mathscr{K}^{\prime}(s)+3 \mathscr{K}^{2}(s)-\mathscr{T}^{2}(s), \\
\delta(s)=3 \mathscr{K}(s) \mathscr{T}(s)+\mathscr{T}^{\prime}(s) .
\end{gathered}
$$

Substituting these into (37), we obtain (35). Conversely if (35) holds, it is easy to show that $\alpha$ is of type AW(2). This completes the proof.

Corollary 16. Let $\alpha: I \rightarrow G_{3}$ be a curve (of osculating order 3 ) in the equiform geometry of the Galilean space $G_{3}$.

(i) If $\alpha$ is an isotropic logarithmic spiral in $G_{3}$, then $\alpha$ is of type $A W(2)$ curve.

(ii) If $\alpha$ is a circular helix in $G_{3}$, then there is not circular helix of type $A W(2)$.

(iii) If $\alpha$ is an isotropic circle in $G_{3}$, then $\alpha$ is of type $A W(2)$ curve.

Theorem 17. Let $\alpha: I \rightarrow G_{3}$ be a curve (of osculating order 3 ) in the equiform geometry of the Galilean space $G_{3}$. If $\alpha$ is of type $A W(3)$, then

$$
3 \mathscr{K}(s) \mathscr{T}(s)+\mathscr{T}^{\prime}(s)=0 .
$$

Proof. Since $\alpha$ curve is of type AW(3), (31) holds on $\alpha$. So substituting (19) and (21) into (31), we have (39). The converse statement is trivial. Hence our theorem is proved.

\section{Bertrand Curves of $\mathbf{A W}(k)$-Type in the Equiform Geometry of $G_{3}$}

This section characterizes the curvatures of AW $(k)$-type Bertrand curves in the equiform geometry of the Galilean space $G_{3}$. We provided some theorems and conclusion to show that there are Bertrand curves of weak AW(2)-type and AW(3)-type in the equiform geometry of the Galilean space $G_{3}$.

Definition 18. A curve $\alpha: I \rightarrow G_{3}$ with $\kappa(s) \neq 0$ is called a Bertrand curve if there exists a curve $\tilde{\alpha}: I \rightarrow G_{3}$ such that the principal normal lines of $\alpha$ and $\widetilde{\alpha}$ at $s \in I$ are equal. In this case $\tilde{\alpha}$ is called a Bertrand mate of $\alpha$ [11]. 
The curve $\tilde{\alpha}$ is called a Bertrand mate of $\alpha$ and vice versa. A Frenet framed curve is said to be a Bertrand curve if it admits a Bertrand mate.

By definition, for a Bertrand pair $(\alpha, \widetilde{\alpha})$, there exists a functional relation $\widetilde{s}=\widetilde{s}(s)$ such that

$$
\tilde{\lambda}(\widetilde{s}(s))=\lambda(s) .
$$

Let $(\alpha, \tilde{\alpha})$ be a Bertrand mate in the equiform geometry of the Galilean space $G_{3}$. Then we can write

$$
\tilde{\alpha}(s)=\alpha(s)+\lambda N(s) .
$$

Theorem 19. Let $(\alpha, \tilde{\alpha})$ be Bertrand mate in the equiform geometry of the Galilean space $G_{3}$. Then the function $\lambda$ defined by relation (41) is a constant, and the equiform curvature $\mathscr{K}(s)=0$.

Proof. Let $\{T(s), N(s), B(s)\}$ and $\{\widetilde{T}(s), \widetilde{N}(s), \widetilde{B}(s)\}$ be the Frenet frames according to the equiform geometry of the Galilean space $G_{3}$ along $\alpha(s)$ and $\widetilde{\alpha}(s)$, respectively. Since $(\alpha(s), \widetilde{\alpha}(s))$ is a Bertrand mate, from (41) we can write

$$
\tilde{\alpha}(s)=\alpha(s)+\lambda N(s) .
$$

By differentiation of (42) with respect to $s$, we obtain

$$
\widetilde{T} \frac{d \widetilde{s}}{d s}=T+\lambda^{\prime} N(s)+\lambda N^{\prime}(s),
$$

where $s$ and $\widetilde{s}$ are parameters on $\alpha$ and $\tilde{\alpha}$, respectively; $(d \widetilde{s} / d s) \neq 0$. By using relation (17) we have

$$
(\widetilde{\alpha}(s))^{\prime}=\widetilde{T} \frac{d \widetilde{s}}{d s}=T(s)+\left(\lambda^{\prime}+\lambda \mathscr{K}(s)\right) N(s)+\lambda \mathscr{T}(s) B(s) .
$$

Since $(\widetilde{\alpha}(s))^{\prime}$ is parallel to $\widetilde{T}(s)$, then

$$
(\widetilde{\alpha}(s))^{\prime} \perp \widetilde{N}(s) .
$$

Since $\widetilde{N}(s)$ is parallel to $N(s)$, then

$$
(\widetilde{\alpha}(s))^{\prime} \perp N(s) .
$$

Thus, from (45) and (46), we have

$$
\left\langle(\widetilde{\alpha}(s))^{\prime}, N(s)\right\rangle=0 .
$$

Substituting (44) into (47), we obtain

$$
\lambda^{\prime}+\lambda \mathscr{K}(s)=0 .
$$

Thus, from (48), we get that $\lambda$ is constant, and $\mathscr{K}(s)=0$. Hence, the proof is completed.

Theorem 20. Let $(\alpha, \tilde{\alpha})$ be Bertrand mate in the equiform geometry of the Galilean space $G_{3}$. Then angle measurement of this curve between tangent vectors at corresponding points is constant.
Proof. If we show $\langle\widetilde{T}(s), T(s)\rangle^{\prime}=0$, then the proof is complete:

$$
\begin{aligned}
\langle\widetilde{T}(s), T(s)\rangle^{\prime}= & \left\langle(\widetilde{T}(s))^{\prime}, T(s)\right\rangle+\left\langle\widetilde{T}(s),(T(s))^{\prime}\right\rangle \\
= & \langle\widetilde{\mathscr{K}}(s) \widetilde{T}(s)+\widetilde{N}(s), T(s)\rangle \\
& +\langle\widetilde{T}(s), \mathscr{K}(s) T(s)+N(s)\rangle \\
= & \widetilde{\mathscr{K}}(s)\langle\widetilde{T}(s), T(s)\rangle+\langle\widetilde{N}(s), T(s)\rangle \\
& +\mathscr{K}(s)\langle\widetilde{T}(s), T(s)\rangle \\
& +\langle\widetilde{T}(s), N(s)\rangle .
\end{aligned}
$$

Since $\widetilde{N}(s)$ is parallel to $N(s)$ and $N(s) \perp T(s)$, then

$$
\langle\widetilde{N}(s), T(s)\rangle=0 .
$$

Since $\widetilde{N}(s)$ is parallel to $N(s)$ and $\widetilde{T}(s) \perp \widetilde{N}(s)$, then

$$
\langle\widetilde{T}(s), N(s)\rangle=0 .
$$

Since $(\alpha, \widetilde{\alpha})$ is Bertrand mate in the equiform geometry of the Galilean space $G_{3}$, from Theorem 19 we have

$$
\mathscr{K}(s)=0, \quad \widetilde{\mathscr{K}}(s)=0 .
$$

So, substituting (50), (51), and (52) into (49), we have

$$
\langle\widetilde{T}(s), T(s)\rangle^{\prime}=0 .
$$

Hence, the proof is completed.

Theorem 21. Let $\alpha: I \rightarrow G_{3}$ be a curve in the equiform geometry of the Galilean space $G_{3}$. Then $\alpha$ is a Bertrand curve if and only if $\alpha$ is a curve with constant torsion $\mathscr{T}(s)$.

Proof. Deneote the Frenet frames of $\alpha(s)$ and $\widetilde{\alpha}(s)$ by $\{T(s), N(s), B(s)\}$ and $\{\widetilde{T}(s), \widetilde{N}(s), \widetilde{B}(s)\}$, respectively. Let angle between $T(s)$ and $\widetilde{T}(s)$ which is tangent vector of $\widetilde{\alpha}(s)$ be $\theta$. As $(N(s), \widetilde{N}(s))$ is a linearly dependent set, we can write

$$
\widetilde{T}(s)=\cos \theta T(s)+\sin \theta B(s) .
$$

If we differentiate (54) and consider $(N(s), \widetilde{N}(s))$ is a linearly dependent set, we can easily see that $\theta$ is a constant function. Since $\alpha(s)$ and $\widetilde{\alpha}(s)$ are Bertrand curve mates, we have

$$
\tilde{\alpha}(s)=\alpha(s)+\lambda N(s) .
$$

If Differentiating (55) with respect to $s$ and with the help of Theorem 19, we get

$$
\widetilde{T} \frac{d \widetilde{s}}{d s}=T(s)+\lambda \mathscr{T} B(s) .
$$

If we consider (54) and (56), we obtain

$$
\cot \theta \lambda \mathscr{T}(s)=1 .
$$


Taking $v=\cot \theta \lambda$, we get

$$
\mathscr{T}(s)=\frac{1}{v} .
$$

This means that $\mathscr{T}$ is constant. The converse statement is trivial. Hence, theorem is proved.

Corollary 22. Let $\alpha: I \rightarrow G_{3}$ be Bertrand curve in the equiform geometry of the Galilean space $G_{3}$. Then $\alpha$ is a circular helix in $G_{3}$.

Proof. Since $\alpha$ is Bertrand curve in the equiform geometry of the Galilean space $G_{3}$, from Theorems 19 and 21 we have

$$
\mathscr{K}(s)=0, \quad \mathscr{T}(s) \text { is constant. }
$$

Thus, $\alpha$ is a circular helix in $G_{3}$. Hence, theorem is proved.

Theorem 23. Let $\alpha: I \rightarrow G_{3}$ be Bertrand curve in the equiform geometry of the Galilean space $G_{3}$. Then $\alpha$ is a weak $A W(2)$-type or $A W(3)$-type curve.

Proof. Now suppose that $\alpha: I \rightarrow G_{3}$ is Bertrand curve in the equiform geometry of the Galilean space $G_{3}$. Then, from Theorems 19 and 21 we have

$$
\mathscr{K}(s)=0, \mathscr{T}(s) \text { is constant, }
$$

if (60) is substituted into (25) and (39), which completes the proof of the theorem.

Theorem 24. Let $\alpha: I \rightarrow G_{3}$ be Bertrand curve in the equiform geometry of the Galilean space $G_{3}$. Then $\alpha$ is not a weak $A W(3)$-type or $A W(2)$-type curve.

Proof. Since $\alpha: I \rightarrow G_{3}$ is Bertrand curve according to the equiform geometry of the Galilean space $G_{3}$, then, (60) holds on $\alpha$. If (60) is substituted in (26) and (35), we get, respectively,

$$
\begin{aligned}
& \mathscr{T}^{2}(s)=0, \\
& \mathscr{T}^{3}(s)=0 .
\end{aligned}
$$

Since $\mathscr{T}$ is nonzero constant, this is impossible. So, $\alpha$ is not a weak AW(3)-type or type AW(2) curve. Hence, the theorem is proved.

\section{Conflict of Interests}

The authors declare that there is no conflict of interests regarding the publication of this paper.

\section{References}

[1] I. M. Yaglom, A Simple Non-Euclidean Geometry and Its Physical Basis, Springer, New York, NY, USA, 1979.

[2] B. J. Pavković and I. Kamenarović, "The equiform differential geometry of curves in the Galilean space $G_{3}$," Glasnik Matematički, vol. 22, no. 2, pp. 449-457, 1987.
[3] B. J. Pavković, "Equiform geometry of curves in the isotropic spaces $I_{3}^{(1)}$ and $I_{3}^{(2)}$," Rad Jugoslavenske Akademije Znanosti $i$ Umjetnosti, no. 421, pp. 39-44, 1986.

[4] C. Özgür and F. Gezgin, "On some curves of $A W(k)$-type," Differential Geometry-Dynamical Systems, vol. 7, pp. 74-80, 2005.

[5] K. Arslan and C. Özgür, "Curves and surfaces of $A W(k)$ type," in Geometry and Topology of Submanifolds, IX (Valenciennes/Lyon/Leuven, 1997), pp. 21-26, World Science, River Edge, NJ, USA, 1999.

[6] M. Külahcı and M. Ergüt, "Bertrand curves of AW(k)-type in Lorentzian space," Nonlinear Analysis. Theory, Methods \& Applications, vol. 70, no. 4, pp. 1725-1731.

[7] S. Kiziltuğ and Y. Yayl, "Bertrand Curves of AW(k)-type in three dimensional lie groups," submitted.

[8] M. Külahcı, A. O. Öğrenmiş, and M. Ergüt, "New characterizations of curves in the Galilean space $G_{3}$," International Journal of Physical and Mathematical Sciences, vol. 1, no. 1, 2010.

[9] S. Kızıltuğ and Y. Yaylı, "On the quaternionic Mannheim curves of AW(k)-type in Euclidean space $E^{3}$," Kuwait Journal of Science. In press.

[10] Z. Erjavec and B. Divjak, "The equiform differential geometry of curves in the pseudo-Galilean space," Mathematical Communications, vol. 13, no. 2, pp. 321-332, 2008.

[11] S. Izumiya and N. Takeuchi, "Generic properties of helices and Bertrand curves," Journal of Geometry, vol. 74, no. 1-2, pp. 97109, 2002. 


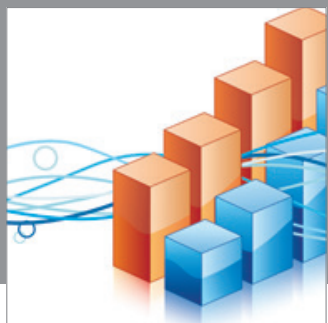

Advances in

Operations Research

mansans

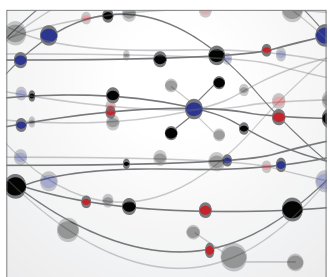

The Scientific World Journal
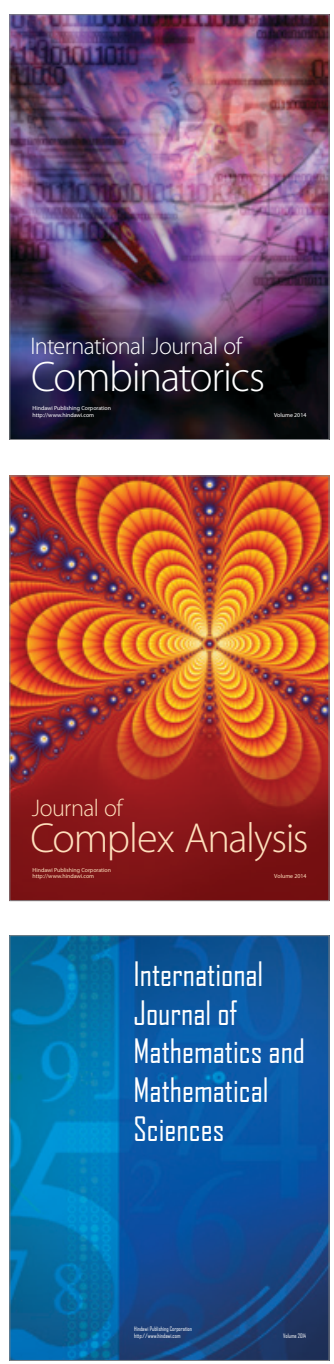
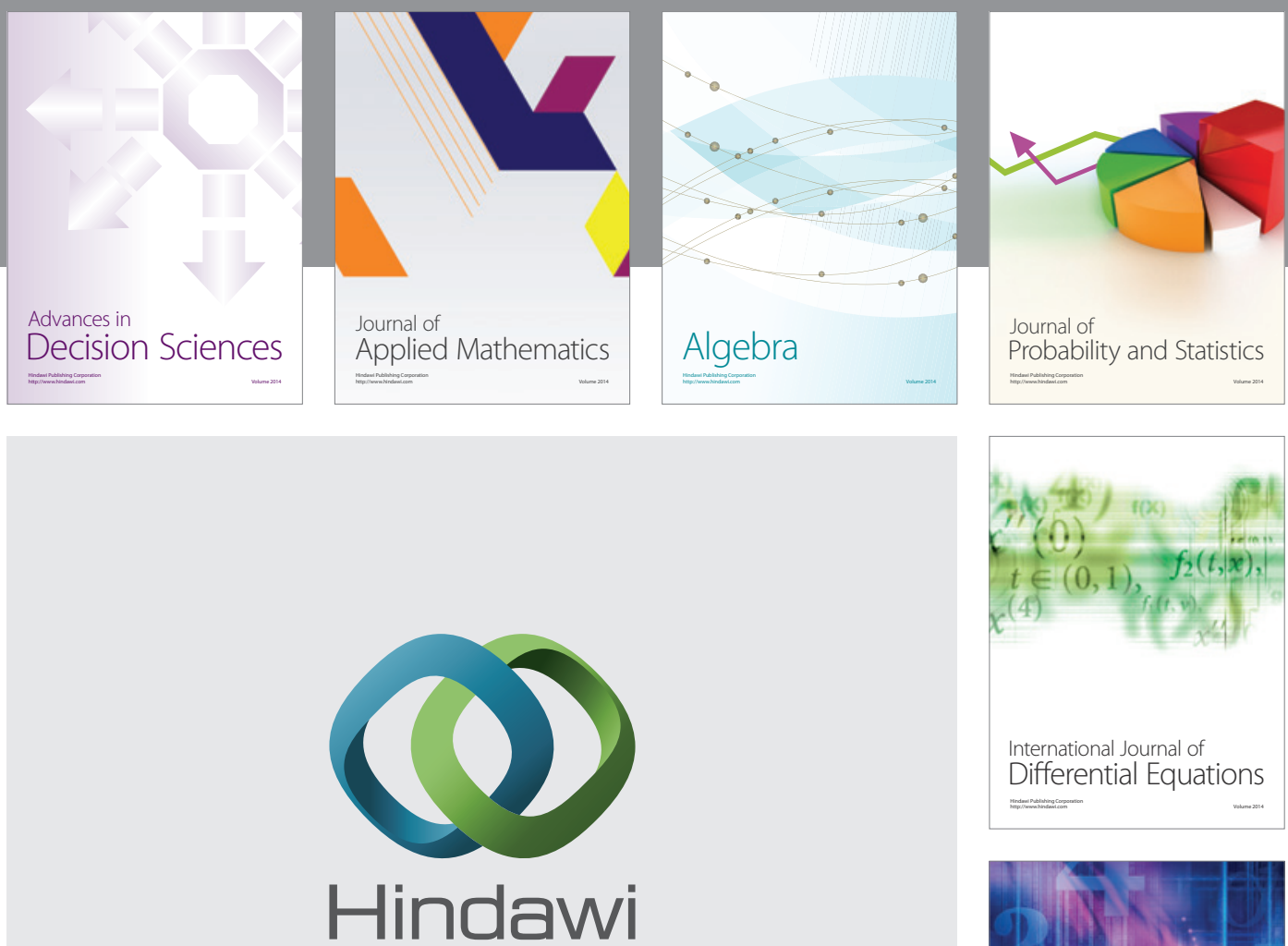

Submit your manuscripts at http://www.hindawi.com
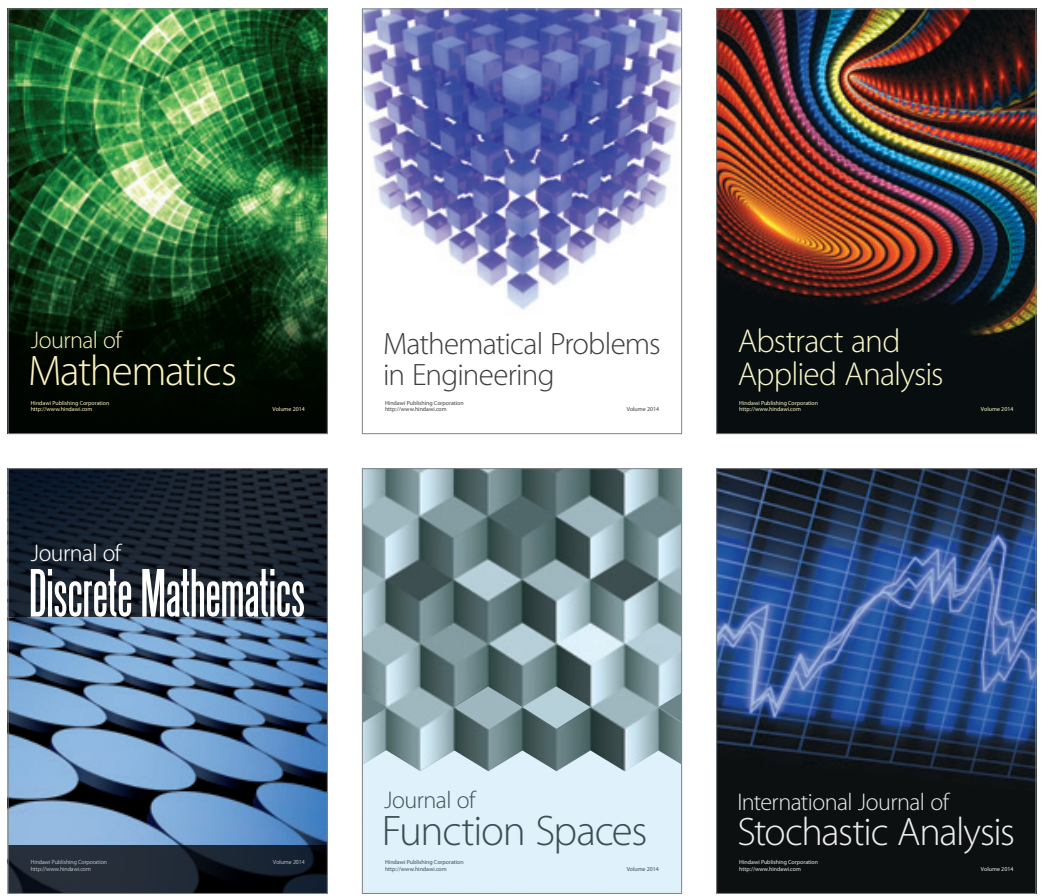

Journal of

Function Spaces

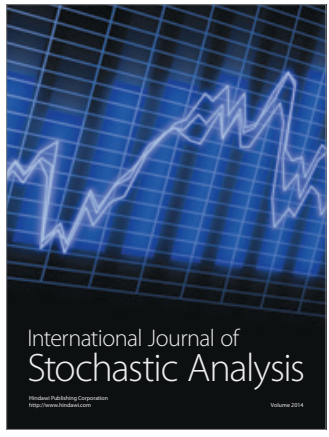

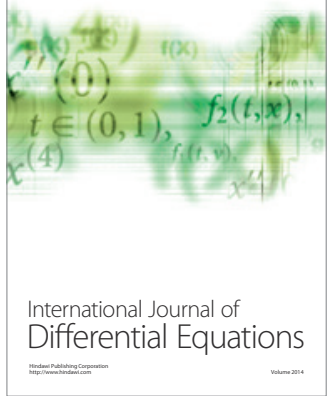
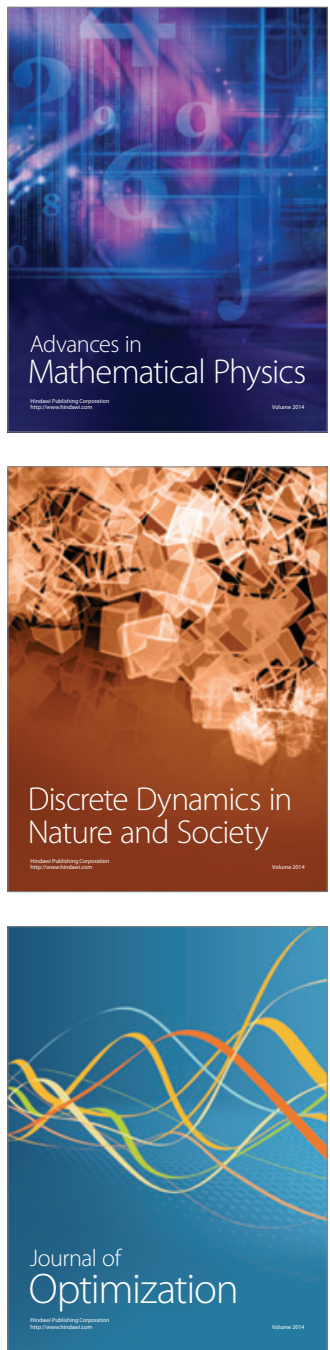